\title{
Abnormal Colonization of Neonates in an Intensive Care Unit: Means of Identifying Neonates at Risk of Infection
}

\author{
KATHERINE SPRUNT, GRACE LEIDY, AND WINIFRED REDMAN \\ Division of Infectious Diseases, The Babies Hospital, The Children's Medical and Surgical Center of New York and \\ the Department of Pediatrics, College of Physicians and Surgeons, Columbia University, New York City, \\ New York, USA
}

\section{Summary}

The current investigation was designed to select infants in the neonatal intensive care unit (ICU) at highest risk of infection. The data, derived from a prospective study of 223 neonates, indicate that neonates in an ICU develop their initial aerobic pharyngeal flora according to one of the three patterns shown in Table 1, one of which (low titer or no growth) is dependent on antibiotic therapy. Of particular importance is the finding that infants with normal flora ( $\alpha$-streptococci predominating) or cultures showing low titers $\left(<10^{4}\right.$ colony-forming units $\left.(\mathrm{cfu}) / \mathrm{ml}\right)$ or no growth did not become infected. All infections occurred in the group of infants with abnormal pharyngeal colonization (18 infections in 115 abnormally colonized infants): with a single exception the infecting organism showed the cultural characteristics of the colonizing organism. Consequently one can pinpoint the relatively small number of infants at particular risk of infection and predict what the infecting organism will be if infection occurs.

\section{Speculation}

These findings indicate indirectly that normal bacterial flora provides a significant host defense mechanism against infection. It seems reasonable that artificial development of "normal" flora by implantation of key constituents ( e.g., $\alpha$-streptococci) in the pharynx should increase the resistance of abnormally colonized high risk infants to infection.

Infection, most of which is nosocomial, is recognized as a significant cause of morbidity and mortality among patients in ICUs. The current investigation was designed to select infants in the neonatal ICU at highest risk of infection and to reduce this risk. The two basic premises to be tested were: 1) that most bacterial infections originating in the ICU develop following high titer abnormal colonization of the pharynx with potential pathogens (i.e., are superinfections) and 2) that acquisition and maintenance of normal pharyngeal bacterial flora provides a host defense mechanism against infection.

The prospectively collected data to be presented show that bacterial superinfection, or clinical infection occurring in infants with abnormal colonization of the pharynx, was the major variety of infection found in the study group of 223 infants 3 days old or older. In this study population only infants with prior or concomitant abnormal bacterial colonization of the pharynx became infected: with a single exception infection was caused by an organism with the cultural characteristics of the abnormal colonizer. Because no infants with normal pharyngeal colonization showed clinical evidence of infection, the data presented support the premise that normal bacterial flora provide a host defense mechanism against infection.

\section{MATERIALS AND METHODS}

\section{SELECTION OF PATIENTS}

Patients 3 or more days of age admitted to the Babies Hospital Neonatal Intensive Care Unit between August 1971 and December 30, 1974 made up the study population. Since there was no recognized bias in patient selection, the study population should be representative of the ICU population as a whole.

\section{CULTURES}

Cultures of the oropharynx were taken at least twice weekly beginning with the first few days of life and continuing when possible until normal flora became established. With rare exceptions all throat cultures were obtained by one person (K.S.) using a nasopharyngeal swab on a flexible wire applicator (Calgiswab). The swab was wiped gently on the posterior pharynx, placed in 1 $\mathrm{ml}$ Trypticase soy broth (BBL), and taken promptly to the laboratory.

Most swabs were wiped across the patients' posterior pharynx in fairly standard fashion. A few, however, usually in patients with precarious respiratory apparatus and paralyzed gag reflex, did not reach beyond the anterior pharyngeal pillars. Simultaneous cultures from the posterior pharynx and anterior pillars were compared in 10 neonates. There was good agreement for numbers of colony-forming units and for the varieties of bacteria found. There was but one exception to dominance of a particular variety of organism. This difference would not have changed the culture's classification pattern (see Definitions below).

\section{PLATING}

The broth containing the swab was mixed on a Vortex Jr. mixer for a few seconds, a smear for gram stain was made and the swab discarded. The residual broth was considered the undiluted culture material.

According to the absence or presence and concentration of organisms in the smear, appropriate dilutions of culture material were plated with calibrated loops $(0.01$ and $0.001 \mathrm{ml})$ on Trypticase soy agar (BBL) containing 5\% horse blood with and without 0.2 , 0.5 , and $1.0 \mu \mathrm{g} / \mathrm{ml}$ of penicillin or ampicillin (8) on mitis-salivarius agar with Tellurite, mannitol salt agar, bile esculin agar, and MacConkey agar (all from Difco). The plates were incubated in $5 \% \mathrm{CO}_{2}$ in air for 20-24 hr (mannitol salt in air) and estimates of colony-forming units of each type of organism recorded. Bacteria were identified according to standard bacteriologic procedures. The highest concentration plated was $0.05 \mathrm{ml}$ of the undiluted culture material.

Definitions: The following broad and arbitrary definitions were adopted. They are based on total colony-forming units and the proportion of types of bacteria comprising the population. 
Table 1. Colonization patterns of oropharyngeal bacterial flora of 223 ICU neonates: Relation of pattern to antibiotic treatment and infection

No. of neonates with pattern and antibiotic treatment

\begin{tabular}{|c|c|c|c|c|}
\hline \multirow{3}{*}{$\begin{array}{l}\text { Colonization } \\
\text { pattern }\end{array}$} & \multirow{2}{*}{\multicolumn{2}{|c|}{ No antibiotic $\mathrm{Rx}$}} & & \\
\hline & & & \multicolumn{2}{|c|}{ During antibiotic $\mathrm{Rx}$} \\
\hline & Number & Infected & Number & Infected \\
\hline Normal & $39(68 \%)$ & 0 & 3 & 0 \\
\hline Low titer or no growth & 0 & 0 & $114(68 \%)$ & 0 \\
\hline High titer abnormal & $18(32 \%)$ & 7 & $49(30 \%)$ & 7 \\
\hline Gram(-)bacilli $^{1}$ & 7 & 5 & 23 & 2 \\
\hline S. aureus & 5 & 2 & 4 & 2 \\
\hline S. epidermidis & 4 & 0 & 14 & 2 \\
\hline Other ${ }^{2}$ & 2 & 0 & 8 & $1^{3}$ \\
\hline \multirow[t]{3}{*}{ Total } & 57 & 7 & 166 & 7 \\
\hline & & $(12 \%$ of 57$)$ & & $(4 \%$ of 166$)$ \\
\hline & & $(39 \%$ of 18$)$ & & $(14 \%$ of 49$)$ \\
\hline
\end{tabular}

\footnotetext{
${ }^{1}$ Gram(-)bacilli: enteric and pseudomonads.

${ }^{2}$ Other (no.): hemolytic Haemophilus, two species (1); Neisseria (1); F. meningosepticum (1) H. parainfluenzae (3); enterococci (2); yeast (2).

${ }^{3}$ F. meningosepticum.
}

"Normal" bacterial flora of the oropharynx contains $\alpha$-hemolytic streptococci as the predominant organism in quantities exceeding $10^{4} \mathrm{cfu} / \mathrm{ml}$ of a culture sample. Other organisms such as nonhemolytic streptococci, Neisseria, and Haemophilus species may be present but $\alpha$-hemolytic streptococci make up the majority of the total population to meet the definition of "normal" flora.

"No growth or low titer" colonization indicates cultures showing no growth from $0.05 \mathrm{ml}$ undiluted culture material or total bacterial populations in titers $<10^{4} \mathrm{cfu} / \mathrm{ml}$.

"High titer abnormal" colonization or bacterial "overgrowth" indicates cultures in which the predominant organism (other than $\alpha$-streptococci) is present in concentrations of $10^{4}$ or greater $\mathrm{cfu} / \mathrm{ml}$ and is generally $>90 \%$ of a sample population. This latter definition is modified for Staphylococcus aureus. Reasons for the modification are noted in context in Results.

"Infection" indicates clinical disease; "superinfection" refers to infection caused by a colonizing organism.

\section{DIAGNOSIS OF INFECTION}

Diagnosis of infection was made by the clinicians caring for the infant, or, in a few instances, by the pathologist at postmortem examination. Cultures documenting the infection were sent by the clinician or pathologist to the Babies Hospital Diagnostic Bacteriology Laboratory. The results were retrieved from the Diagnostic Laboratory records. The infants' charts were reviewed for evidence of clinical compatibility with the Diagnostic Laboratory findings and the clinician or pathologist consulted before the infant was accepted as infected.

\section{RESULTS}

\section{THREE BASIC COLONIZATION PATTERNS}

ICU neonates developed their initial oropharyngeal bacterial flora according to the three basic patterns, "normal," "low titer or no growth," and "high titer abnormal" colonization in the frequencies shown in Table 1 . The "low titer or no growth" pattern occurred only in patients receiving antibiotics.

It is noteworthy that high abnormal colonization occurred in about a third of the infants irrespective of antibiotic treatment. Three infants acquired normal flora $(\alpha$-streptococci predominating) while receiving customary doses of antibiotics including penicillin. These infants are discussed below.

\section{INTERRELATIONSHIPS BETWEEN COLONIZATION PATTERN AND INCIDENCE OF INFECTION}

Infants Not Receiving Antibiotics. The data in Table 1 show that the neonates not receiving antibiotics developed within the first few days of life either normal bacterial flora (68\%) or high titer abnormal colonization with potential pathogens (32\%). There was no evidence of clinical infection in the infants who developed normal flora. All infections occurred in infants with high titer abnormal colonization and were in every instance caused by an organism with the cultural characteristics of the colonizing strain.

The common abnormal colonizing organisms were gram negative enteric type bacilli, Staphylococcus aureus and Staphylococcus epidermidis. As shown in Table 1 the infection rate in abnormally colonized infants was high $(7 / 18)$, and especially so in those colonized by gram-negative bacilli or $S$. aureus (7/12).

Infants Receiving Antibiotics. The antibiotics used in the study interval were initially penicillin and kanamycin, and later penicillin or ampicillin and kanamycin or gentamicin.

Most of the infants receiving antibiotics developed the low titer or no detectable colonization pattern $(68 \%)$ irrespective of whether or not they had acquired some bacterial flora before therapy was started. As shown in Table 1, no infant with the low titer or no growth colonization pattern became infected while on therapy nor did the three infants who developed normal flora while receiving antibiotics. All infections occurred in infants with high abnormal colonization: with a single exception to be discussed subsequently all infections were caused by an organism with the characteristics of the abnormal colonizing strain.

Infants in the "no growth or low titer" colonization group usually developed normal flora or high titer abnormal colonization within a week following cessation of therapy. Ninety-three of the 114 infants in this category could be followed long enough for their post therapy colonization pattern to be documented. Fiftytwo percent (48/93) developed high titer abnormal colonization; $48 \%$ (45/93) developed normal flora (Table 2). Again, no infection was noted in infants who developed normal flora. All infections occurred in those with abnormal colonization and were in each instance due to the organism causing abnormal colonization.

Review of the data from Tables 1 and 2 shows the correlation of infection with high titer abnormal colonization (18 infections in 115 abnormally colonized infants) and the absence of infection in infants with normal flora or with low titer colonization while in that state (none in 201 infants).

\section{DEVELOPMENT OF NORMAL FLORA BY INFANTS RECEIVING ANTIBIOTICS}

Three infants developed and maintained normal flora while receiving antibiotic therapy. By definition this means that $\alpha$-streptococci in high titer predominated in their throat cultures in spite of intravenous penicillin in two infants $(50,000-60,000$ $\mathrm{U} / \mathrm{kg}$ ) and ampicillin in the third (approximately $100 \mathrm{mg} / \mathrm{kg}$ ). The first infant's $\alpha$-streptococci grew on agar containing $5 \mathrm{U}$ 
penicillin $/ \mathrm{ml}$ but not on agar containing $10 \mathrm{U} / \mathrm{ml}$. Approximately half the $\alpha$-streptococci from cultures from the second baby grew on agar containing $1 \mathrm{U}$ but not on agar containing $5 \mathrm{U} / \mathrm{ml}$ of penicillin. The more sensitive streptococci $(<0.5 \mathrm{U} / \mathrm{ml})$ persisted as roughly half the streptococcal population through the 11 th day of life, 3 days after therapy was discontinued. No streptococci in the cultures of the third infant grew on agar containing $0.5 \mu \mathrm{g} / \mathrm{ml}$ ampicillin. Unfortunately the lower limits of resistance were not determined.

\section{ORGANISMS CAUSING HIGH ABNORMAL COLONIZATION AND INCIDENCE OF INFECTION}

The data of Table 3 show the number of patients abnormally colonized with various organisms and the incidence of infection in each group. Gram-negative enteric bacilli and $S$. epidermidis were the most common abnormal colonizers (35\% and $32 \%$, respectively) with $S$. aureus (19\%) the third most common.

$S$. aureus, however, caused a disproportionate number of infections in comparison to the others. Six of the 14 infants shown in Tables 1 and 2 as colonized by $S$. aureus became infected, a higher proportion than the 7 of 43 infants colonized by gram-negative bacilli. These figures for $S$. aureus may be somewhat misleading, however, for reasons noted below.

\section{ABNORMAL OROPHARYNGEAL COLONIZATION BY STAPHYLOCOCCUS AUREUS}

Judged by the colonization of infected babies, "abnormal" colonization by $S$. aureus seems to differ from high titer abnormal colonization by the other types of bacteria considered. "High titer abnormal" colonization by other potential pathogens indicates gross predominance of the potential pathogens ( $>90 \%$ of the population in $82 \%$ of instances) in titers $>10^{4} \mathrm{cfu} / \mathrm{ml}$; assays observed were between $10^{5}$ and $10^{8} \mathrm{cfu} / \mathrm{ml}$, usually $10^{6}-10^{7}$. If a second organism were present at $>10^{4} \mathrm{cfu} / \mathrm{ml}$, it was with few exceptions a second gram-negative bacillus. $\alpha$-Hemolytic streptococci were found in only $11 \%$ and were with one exception $<10 \%$ of the population sample.

Four of the six infants infected with $S$. aureus did not fit our criteria of abnormal colonization. One infant with an assay value of $3 \times 10^{3} \mathrm{cfu} S$. aureus and $8 \times 10^{3} \alpha$-streptococci $/ \mathrm{ml}$ developed $S$. aureus pneumonia. The other three infants had assay values of $>10^{4} \mathrm{~S}$. aureus $/ \mathrm{ml}$ culture samples but they were not predominant $(29 \%, 17 \%$, and $8 \%)$. Alpha streptococci were predominant in one (84\%), S. epidermidis in another (83\%), and a mixed population, including $36 \% \alpha$-streptococci, in the third. Of 10 infants meeting our criteria of abnormal colonization, 2 were infected. The 4 noted above, not in accord with the definition, were added on the basis of an infection.

Table 2. Colonization pattern after cessation of antibiotic treatment for $93^{1}$ infants with low titer or no growth during antibiotic treatment

\begin{tabular}{lll}
\hline $\begin{array}{c}\text { Colonization } \\
\text { pattern } \\
\text { after } \\
\text { antibiotics } \\
\text { discontinued }\end{array}$ & $\begin{array}{c}\text { No. } \\
\text { infants }\end{array}$ & \multicolumn{1}{c}{$\begin{array}{c}\text { No. } \\
\text { infected }\end{array}$} \\
\hline Normal & $45(48 \%)$ & 0 \\
High abnormal & 13 & 0 \\
$\quad$ Gram(-)bacilli & 5 & 2 \\
S. aureus & 22 & 1 \\
S. epidermidis & $\underline{8}$ & $\underline{1^{3}}$ \\
Other & & $4(8 \%$ of 48$)$ \\
Total & $48(52 \%)$ & $4(4 \%)$ \\
Overall total & 93 &
\end{tabular}

\footnotetext{
${ }^{1}$ Twenty-one (18\%) of 114 infants in the group had inadequate followup cultures.

${ }^{2}$ Other (no.): Neisseria (2); yeast (3); H. parainfluenzae (1); nonhemolytic streptococcus (1); group B streptococcus (1).
}

Table 3. Organisms causing high titer abnormal colonization and

\begin{tabular}{|c|c|c|}
\hline Colonizing organism & $\begin{array}{c}\text { Total } \\
\text { patients }\end{array}$ & $\begin{array}{c}\text { No. } \\
\text { infected }\end{array}$ \\
\hline Gram(-)bacilli-E. coli & 23 & 5 \\
\hline Klebsiella sp. & 15 & \\
\hline Pseudomonas sp. & 4 & 1 \\
\hline Proteus mirabilis & 1 & $\underline{1}$ \\
\hline Total & $43(35 \%)$ & 7 \\
\hline S. epidermidis & $40(32 \%)$ & 3 \\
\hline S. aureus & $23(19 \%)$ & 6 \\
\hline \multicolumn{3}{|l|}{ Other } \\
\hline Neisseria sp. & 3 & \\
\hline $\begin{array}{l}\text { Flavobacterium } \\
\text { meningosepticum }\end{array}$ & 1 & 1 \\
\hline Enterococcus & 2 & \\
\hline Yeast & 5 & \\
\hline Haemophilus sp. & 5 & \\
\hline $\begin{array}{l}\text { Nonhemolytic } \\
\text { streptococcus }\end{array}$ & 1 & \\
\hline Group B Streptococcus & 1 & $\underline{1}$ \\
\hline Total & $18(14 \%)$ & 2 \\
\hline Total & 124 & $18(15 \%)$ \\
\hline
\end{tabular}

If all infants were reconsidered for abnormal colonization by $S$. aureus on the basis of less stringent requirements $\left(10^{3} \mathrm{cfu} / \mathrm{ml}\right.$ or more and not necessarily the predominant organism, but greater than $2 \%$ of the total population) 9 additional babies can be added, giving the total of 23 shown in Table 3 . It is evident that abnormal colonization of the oropharynx with $S$. aureus is still relatively infrequent (19\%), but the infection rate for colonized infants was high $(26 \%)$.

\section{INFECTED INFANTS}

The 18 infected infants (Table 3 ) are listed in Table 4 according to type of infection, organism causing the infection, and use of antibiotics before the infection.

Eight of the 18 infected infants had demonstrated bacteremia with one or more positive blood cultures and compatible clinical findings. One of the infants listed as having $S$. epidermidis sepsis had multiple positive blood cultures. The other, an infant with respiratory distress syndrome (RDS) is recorded in this group because of a single blood culture positive for $S$. epidermidis and a compatible clinical picture. This infant also had clinically evident omphalitis apparently due to $S$. aureus. His pharyngeal cultures showed abnormal colonization by $S$. epidermidis and no $S$. aureus was noted, but type $52 S$. aureus grew from pus from a red and indurated umbilicus. This infant is the exception referred to previously.

An additional infant is included in the "sepsis" group though his sepsis was undocumented. This infant was recovering from RDS when his oropharynx became heavily colonized with "pure" cultures of Flavobacterium meningosepticum following contamination of his respiratory apparatus with this organism. Shortly thereafter he became irritable, lethargic, his respiratory rate increased, his general condition deteriorated rapidly, and he was considered clinically to be septic. His single blood culture taken while on antibiotics showed no growth. His is the only undocumented infection accepted as such.

Two of the infants with $S$. aureus sepsis had a probable peripheral source of infection in that each had a hyperalimentation line shown to be contaminated with $S$. aureus. Both infants had high titers of $S$. aureus in the pharynx, but it was predominant in neither. $\alpha$-Hemolytic streptococci were present in both and predominant in one.

Another infant with sepsis who was abnormally colonized by Escherichia coli from day four on was clinically well when $E$. coli was grown from the tip of the umbilical artery catheter removed on his 5th day. He was febrile on day seven and blood stream invasion was documented on the 10th and 14th days of life. 
Pyarthrosis and osteomyelitis were documented on the 15th day.

The sepsis caused by group B $\beta$-hemolytic streptococci was clearly nosocomial. The infant had been carefully followed with biweekly throat cultures. Group B streptococci were first noted and were in virtually pure culture on his 46th day; his blood culture was positive on his $53 \mathrm{rd}$ day.

Both of the infants with staphylococcal pneumonia diagnosed clinically and by $\mathrm{x}$-ray were thought to be septic. Only one had a blood culture taken at a pertinent time and it was negative. The diagnosis of $E$. coli pneumonia in the third infant was made at postmortem examination following death on the 11th day from severe RDS and pulmonary dysplasia. His study cultures on days 3,6 , and 10 had grown apparently pure $E$. coli in titers of $2 \times$ $10^{4}-4 \times 10^{5} / \mathrm{ml}$. A culture of nasotracheal aspirate sent to the diagnostic laboratory on day 10 showed heavy growth of $E$. coli. His postmortem blood culture showed the same organism.

The diagnosis of omphalitis was based on erythema, induration, and pus from the umbilicus.

The infant with mediastinitis had severe RDS, pneumothoraces, and indwelling chest tubes. Only $S$. epidermidis was recovered from his pharynx until just before death on the eighth day of life when $\alpha$-streptococci were beginning to appear. Cultures sent to the diagnostic laboratory before death showed $S$. epidermidis from the chest drainage tube and nasotracheal tube. Only $S$. epidermidis

Table 4. Infected infants by type of infection, antibiotic experience, and infecting organism

\begin{tabular}{|c|c|c|c|c|}
\hline \multirow[b]{2}{*}{$\begin{array}{l}\text { Type of } \\
\text { infection }\end{array}$} & \multicolumn{2}{|c|}{ Antibiotic $\mathrm{Rx}$} & \multicolumn{2}{|c|}{ No antibiotic $\mathrm{Rx}$} \\
\hline & Organism & $\begin{array}{l}\text { No. } \\
\text { in- } \\
\text { fants }\end{array}$ & Organism & $\begin{array}{l}\text { No. } \\
\text { in- } \\
\text { fants }\end{array}$ \\
\hline \multirow[t]{4}{*}{ Sepsis } & S. epidermidis & 2 & E. coli & 2 \\
\hline & S. aureus & 1 & S. aureus & 2 \\
\hline & $\begin{array}{l}\text { F. meningo- } \\
\text { septicum }^{1}\end{array}$ & 1 & & \\
\hline & $\begin{array}{l}\text { Group B } \\
\text { streptococcus }\end{array}$ & 1 & & \\
\hline \multirow[t]{2}{*}{ Pneumonia } & S. aureus & 2 & & \\
\hline & E. coli & 1 & & \\
\hline \multirow[t]{2}{*}{$U T I$} & & & E. coli & 2 \\
\hline & & & $\begin{array}{l}\text { Proteus } \\
\quad \text { mirabilis }\end{array}$ & 1 \\
\hline Omphalitis & S. aureus & 1 & & \\
\hline Mediastinitis & S. epidermidis & 1 & & \\
\hline $\begin{array}{l}\text { Tracheobronchitis } \\
\text { and ophthalmitis }\end{array}$ & P. aeruginosa & 1 & & \\
\hline
\end{tabular}

grew from a rectal swab. Blood and CSF cultures showed no growth. The tip of the chest tube was found in the mediastinum at autopsy; the area around it showed signs of infection. The postmortem lung culture yielded $S$. epidermidis and the blood culture both $S$. epidermidis and Pseudomonas maltophilia.

The infant with tracheobronchitis and an eye infection was severely incapacitated with RDS, necessitating long term use of endotracheal tubes and respiratory support. He became colonized with apparently pure cultures of Pseudomonas aeruginosa and was included as an infected infant when he developed presumed tracheitis and a florid eye infection due to $P$. aeruginosa. Endotracheal tubes removed from his trachea were shaggy with green growth from which pseudomonas organisms were grown in high titer.

It should be noted that an infected or an abnormally colonized infant is counted only once although some, after treatment for one infection, became colonized with another potential pathogen and were subsequently infected by it. One of the infants abnormally colonized by $E$. coli and being treated for an $E$. coli urinary tract infection, for instance, became abnormally colonized during therapy by a Klebsiella species. Within a week he developed a urinary tract infection with this organism. The most dramatic example is that of one infant with omphalitis due to $S$. aureus. His pharyngeal $S$. aureus was replaced during oxacillin therapy by high titer colonization with $P$. aeruginosa and he developed a wound infection, cultures of which yielded heavy growth of pseudomonas. After therapy for the pseudomonas infection, his throat cultures showed high abnormal colonization by $S$. epidermidis; nasotracheal tubes, the site of entry of his hyperalimentation central venous line and on two occasions his blood yielded $S$. epidermidis.

\section{SIGNIFICANT CHARACTERISTICS OF INFECTED INFANTS}

Fifteen of the 18 infected infants were initially diagnosed as having a RDS. Thirteen had hyaline membrane disease (HMD). This raised the question that the diagnosis RDS or HMD included some significant factor favoring infection.

Fifteen of the 18 infants ( 13 with HMD) required some form of mechanical respiratory assistance and all but one infected infant had a indwelling umbilical vessel, usually arterial, catheter. Although figures are not available for incidence of use of these support measures in the ICU population in general, infected babies are believed to have been disproportionately exposed to these obvious hazards.

Since a large proportion of infants with HMD required umbilical artery catheterization and respiratory assistance, an attempt was made within the confines of the study population to see whether something inherent in infants with HMD was playing a significant role, or whether the therapy required for these infants was of major importance. The correlation between diagnosis, antibiotic therapy, culture pattern, infection, and the factors re-

Table 5. Correlation between diagnosis, high risk therapy, antibiotic exposure, culture pattern, and infection

\begin{tabular}{|c|c|c|c|c|c|c|c|}
\hline \multirow[b]{3}{*}{$\begin{array}{l}\text { Antibiotic } \\
\text { category }\end{array}$} & \multirow[b]{3}{*}{ High risk therapy } & \multicolumn{6}{|c|}{ Culture pattern of 223 infants with HMD or other diagnoses } \\
\hline & & \multicolumn{3}{|c|}{ HMD } & \multicolumn{3}{|c|}{ Other } \\
\hline & & Normal & $\begin{array}{l}\text { Low titer } \\
\text { or } \\
\text { no growth }\end{array}$ & $\begin{array}{c}\text { High } \\
\text { abnormal }\end{array}$ & Normal & $\begin{array}{l}\text { Low titer } \\
\text { or } \\
\text { no growth }\end{array}$ & $\begin{array}{c}\text { High } \\
\text { abnormal }\end{array}$ \\
\hline \multirow[t]{4}{*}{$\mathrm{Rx}$} & $\mathrm{U} / \mathrm{A}$ and/or $\mathrm{U} / \mathrm{V}^{1}$ & 0 & $49 / 55$ & $28 / 29$ & $1 / 3$ & $31 / 59$ & $10 / 20$ \\
\hline & Intubated & 0 & $36 / 55$ & $20 / 29$ & $1 / 3$ & $16 / 59$ & $5 / 20$ \\
\hline & + Pressure $^{2}$ & 0 & $48 / 55$ & $22 / 29$ & $1 / 3$ & $15 / 59$ & $5 / 20$ \\
\hline & Infected & 0 & 0 & $6 / 29$ & 0 & 0 & $1 / 20$ \\
\hline \multirow[t]{4}{*}{ No $\mathrm{Rx}$} & $\mathrm{U} / \mathrm{A}$ and/or U/V & $18 / 22$ & 0 & $11 / 11$ & $8 / 17$ & 0 & $3 / 7$ \\
\hline & Intubated & $4 / 22$ & 0 & $5 / 11$ & $2 / 17$ & 0 & $1 / 7$ \\
\hline & + Pressure & $14 / 22$ & 0 & $9 / 11$ & $2 / 17$ & 0 & 0 \\
\hline & Infected & 0 & 0 & $6 / 11$ & 0 & 0 & $1 / 7$ \\
\hline
\end{tabular}

${ }^{1} \mathrm{U} / \mathrm{A}, \mathrm{U} / \mathrm{V}$ : indwelling umbilical artery or venous catheter.

${ }^{2}+$ Pressure: includes nasal CPAP. 
Table 6. Table 5 correlations applied to $93^{1}$ infants with follow-up after low titer or no growth during antibiotic treatment

\begin{tabular}{|c|c|c|c|c|}
\hline \multirow[t]{2}{*}{ High risk therapy } & \multicolumn{2}{|c|}{ HMD } & \multicolumn{2}{|c|}{ Other } \\
\hline & Normal & $\begin{array}{c}\text { High } \\
\text { abnormal }\end{array}$ & Normal & $\begin{array}{c}\text { High } \\
\text { abnormal }\end{array}$ \\
\hline $\mathrm{U} / \mathrm{A}$ and $/$ or $\mathrm{U} / \mathrm{V}^{2}$ & $12 / 14$ & $26 / 29$ & $19 / 31$ & $13 / 19$ \\
\hline Intubated & $8 / 14$ & $17 / 29$ & $8 / 31$ & $7 / 19$ \\
\hline+ Pressure $^{3}$ & $10 / 14$ & $24 / 29$ & $7 / 31$ & $8 / 19$ \\
\hline Infected & 0 & 1 & 0 & 3 \\
\hline
\end{tabular}

${ }^{1}$ See Table 2.

${ }^{2} \mathrm{U} / \mathrm{A}, \mathrm{U} / \mathrm{V}$ : indwelling umbilical artery or venous catheter.

${ }^{3}+$ Pressure: includes nasal CPAP.

Table 7. Summary of infected infants in relation to high risk factors and HMD or other diagnoses

\begin{tabular}{lccl}
\hline \multicolumn{1}{c}{ High risk therapy } & HMD & Other & Total \\
\hline U/A and/or U/V & $12 / 13$ & $5 / 5$ & $17 / 18$ \\
Intubated & $10 / 13$ & $4 / 5$ & $14 / 18$ \\
+ Pressure & $11 / 13$ & $3 / 5$ & $14 / 18$ \\
\hline
\end{tabular}

lated to care of patients with HMD is shown in Table 5. Similar data for infants with the "low titer or no growth" pattern after antibiotics were discontinued are included in Table 6. Although there is some suggestion that patients with HMD have a higher infection rate than those with other diagnoses $(P<0.1,>0.05)$, the figures are too small to permit a significant differentiation between disease state and its therapy in etiology of infection (Tables 5-7).

Be that as it may, whatever plays a role in determining colonization and subsequent infection, it is the abnormally colonized infant who is significantly at risk of infection. Infection, if it occurs, will most likely be caused by an organism with the cultural characteristics of the colonizing strain.

\section{DISCUSSION}

The data presented indicate that neonates in an ICU develop their initial aerobic pharyngeal flora according to one of the three patterns shown in Table 1, one of which (low titer or no growth) is dependent on antibiotic therapy. Of particular importance is the finding that infants with normal flora ( $\alpha$-streptococci predominating) or cultures showing low titers $\left(<10^{4} \mathrm{cfu} / \mathrm{ml}\right)$ or no growth did not become infected. All infections occurred in the group of infants with abnormal pharyngeal colonization; with a single exception the infecting organism showed the cultural characteristics of the colonizing organism. Consequently, one can pinpoint the relatively small number of infants at particular risk of infection and predict what the infecting organism will be if infection occurs. One this basis we recommend that infants in a neonatal ICU or other high risk situation have routine throat cultures taken two to three times a week until a normal pattern is achieved. Standard, well streaked culture plates can be read as showing light, moderate, and heavy growth of predominant $\alpha$-hemolytic streptococci, for instance, or of some other predominant organism with no or rare $\alpha$-streptococci present. This simple procedure permits selection of those at unusually high risk of infection and allows antibiotic susceptibility testing of the organism with which they may become infected. Abnormally colonized infants can then be followed with particular care with respect to early diagnosis and intelligently directed early therapy.

Abnormal high titer colonization designating the high risk state is clearly defined except with respect to $S$. aureus. It is difficult to understand why this organism alone should behave differently from the others. A possible explanation, however, is that relatively low counts and proportions of $S$. aureus may be due to the characteristic clumping pattern of growth, in which a clump of multiple organisms produces a single colony on a plate.

We do not mean to imply by the data presented that uninhibited growth of potential pathogens in the pharynx is necessarily the source of the infecting organisms. It may be that colonization of the pharynx reflects total body colonization by a single organism or colonization in a potentially more significant site other than the pharynx, such as the gut. Nor do we mean to imply that obvious peripheral sources of infection are not significant. It is probably just fortuitous that the only evidence for this in our data is the "exception," the umbilical infection with $S$. aureus following multiple attempts to insert a catheter in an infant abnormally colonized (and infected) by S. epidermidis. Undescribed episodes have been noted in which iv sites were colonized by infecting organisms, but in each instance the organism has been the same as that colonizing the pharynx. At the moment we can say only that pharyngeal colonization provides a significant key to the risk of infection and probable infecting organism.

Neither do we mean to imply that $\alpha$-streptococci themselves are not potential pathogens. In a sick neonate virtually any organism can play a pathogenic role. Alpha streptococci form the backbone of our definition of "normal" flora on the basis of observation. We have, however, seen infants infected with $\alpha$-streptococci. The possibility that strain differences are significant here exists, but no documentation is available. The data suggest that infection by $\alpha$-streptococci is rarer than infection by $S$. epidermidis, an organism commonly considered part of "normal" flora.

The salient finding to emerge from the study is that not one of the 42 infants who developed normal flora ( $\alpha$-streptococci predominating) became demonstrably infected. It has been recognized for some time that suppression of $\alpha$-streptococci by penicillin therapy is followed by abnormal colonization of the pharynx and the threat of superinfection $(2,4-7)$. Prospective selection of penicillinresistant $\alpha$-streptococci in pharyngeal flora by prior oral administration of penicillin permits survival of the $\alpha$-streptococci during high dose penicillin therapy, and abnormal colonization does not occur (4). The data from the three infants with normal flora in spite of antibiotic therapy indicate that low degrees of resistance of $\alpha$-streptococci to penicillin or ampicillin are sufficient to permit persistence in neonates as well as in older individuals. The evidence suggests that the presence and persistence of $\alpha$-streptococci as predominant organism (our definition of normal flora) provides a host defense mechanism against infection $(1,3)$. This rationale is strengthened by the finding that many strains of $\alpha$-streptococci inhibit growth of common abnormal colonizing strains in vitro as well as they apparently do in vivo. It seems reasonable to pursue these findings to the next logical step, that of artificially inducing "normal" pharyngeal colonization by implanting $\alpha$-streptococci in the nasopharynx of high risk infants to increase their resistance to infection. The feasibility of this approach is under investigation.

\section{REFERENCES AND NOTES}

1. Johanson, W. G., Blackstock, R., Pierce, A. K., and Sanford, J. P.: The role of bacterial antagonism in pneumococcal colonization of the human pharynx. $\mathrm{J}$. Lab. Clin. Med., 75: 946 (1970).

2. Louria, D. B., and Brayton, R. G.: The efficacy of penicillin regimens with observations on the frequency of superinfection. J.A.M.A., 186: 987 (1963).

3. Sanders, E.: Bacterial interference. I. Its occurrence among the respiratory tract flora and characterization of inhibition of Group A streptococci by viridans streptococci. J. Infect. Dis., 120: 698 (1969).

4. Sprunt, K., Leidy, G., and Redman, W. R.: Prevention of bacterial overgrowth. J. Infect. Dis., 123: 1 (1971).

5. Sprunt, K., and Redman, W.: Evidence suggesting importance of role of interbacterial inhibition in maintaining balance of normal flora. Ann. Int. Med., 68: 579 (1968).

6. Tillotson, J. R., and Finland, M.: Bacterial colonization and clinical superinfection of the respiratory tract complicating antibiotic treatment of pneumonia. J. Infect. Dis., 119: 597 (1969).

7. Weinstein, L., Goldfield, M., and Chang, T. W.: Infections occurring during chemotherapy: A study of their type, frequency and predisposing factors. N. Engl. J. Med., 251: 247 (1954).

8. Penicillin or ampicillin included to select $\alpha$-streptococci sufficiently resistant to persist in the uninflamed pharynx of infants receiving these antibiotics.

9. This research was supported by SCOR Grant HL 14218, NHLI.

10. Requests for reprints should be addressed to: Katherine Sprunt, M.D., Box 50, Babies Hospital, 167th St. and Broadway, New York City, NY 10032 (USA). 11. Received for publication September 6, 1977.

12. Accepted for publication January $5,1978$. 